\title{
Cost-Effectiveness of Pembrolizumab Versus Ipilimumab in Ipilimumab-Naïve Patients with Advanced Melanoma in the United States
}

\author{
Jingshu Wang, PhD; Bartosz Chmielowski, MD, PhD; James Pellissier, PhD; \\ Ruifeng Xu, PhD; Kendall Stevinson, MD, MPH; and Frank Xiaoqing Liu, PhD
}

\begin{abstract}
BACKGROUND: Recent clinical trials have shown that pembrolizumab significantly prolonged progression-free survival and overall survival compared with ipilimumab in ipilimumab-naïve patients with unresectable or metastatic melanoma. However, there has been no published evidence on the cost-effectiveness of pembrolizumab for this indication.
\end{abstract}

OBJECTIVE: To assess the long-term cost-effectiveness of pembrolizumab versus ipilimumab in ipilimumab-naïve patients with unresectable or metastatic melanoma from a U.S. integrated health system perspective.

METHODS: A partitioned-survival model was developed, which divided overall survival time into progression-free survival and postprogression survival. The model used Kaplan-Meier estimates of progressionfree survival and overall survival from a recent randomized phase 3 study (KEYNOTE-006) that compared pembrolizumab and ipilimumab. Extrapolation of progression-free survival and overall survival beyond the clinical trial was based on parametric functions and literature data. The base-case time horizon was 20 years, and costs and health outcomes were discounted at a rate of $3 \%$ per year. Clinical data-including progressionfree survival and overall survival data spanning a median follow-up time of 15 months, as well as quality of life and adverse event data from the ongoing KEYNOTE-006 trial-and cost data from public sources were used to populate the model. Costs included those of drug acquisition, treatment administration, adverse event management, and disease management of advanced melanoma. The incremental cost-effectiveness ratio (ICER) expressed as cost difference per quality-adjusted life-year (QALY) gained was the main outcome, and a series of sensitivity analyses were performed to test the robustness of the results.

RESULTS: In the base case, pembrolizumab was projected to increase the life expectancy of U.S. patients with advanced melanoma by 1.14 years, corresponding to a gain of 0.79 discounted QALYs over ipilimumab. The model also projected an average increase of $\$ 63,680$ in discounted perpatient costs of treatment with pembrolizumab versus ipilimumab. The corresponding ICER was $\$ 81,091$ per QALY (\$68,712 per life-year) over a 20 -year time horizon. With $\$ 100,000$ per QALY as the threshold, when input parameters were varied in deterministic one-way sensitivity analyses, the use of pembrolizumab was cost-effective relative to ipilimumab in most ranges. Further, in a comprehensive probabilistic sensitivity analysis, the ICER was cost-effective in $83 \%$ of the simulations.

CONCLUSIONS: Compared with ipilimumab, pembrolizumab had higher expected QALYs and was cost-effective for the treatment of patients with unresectable or metastatic melanoma from a U.S. integrated health system perspective.

J Manag Care Spec Pharm. 2017;23(2):184-94

Copyright $\odot 2017$, Academy of Managed Care Pharmacy. All rights reserved.

\section{What is already known about this subject}

Advanced (unresectable or metastatic) melanoma is a severe and costly condition.

Programmed death 1 (PD-1) inhibitors are effective new immunotherapies for the treatment of patients with advanced melanoma. Pembrolizumab, the first FDA-approved PD-1 inhibitor, is associated with longer progression-free survival and overall survival as compared with ipilimumab.

\section{What this study adds}

Pembrolizumab has the potential to significantly increase qualityadjusted life-years (QALY) in patients with advanced melanoma. Pembrolizumab is projected to be cost-effective in comparison with ipilimumab among ipilimumab-naive patients with advanced melanoma at a willingness to pay of $\$ 100,000$ per QALY from the perspective of a U.S. integrated health system.

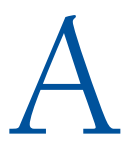
$s$ the first U.S. Food and Drug Administration (FDA)approved anti-programmed cell death-1 (PD-1) immunotherapy, pembrolizumab (KEYTRUDA, Merck \& Co) was found to benefit patients in terms of saving and improving patient lives. Specifically, it was shown to significantly delay disease progression in ipilimumab-refractory and ipilimumab-naive advanced melanoma populations., ${ }^{1,2}$ Also, KEYNOTE-006 (KN006)—a randomized, controlled, open-label, 3-arm pivotal study of 2 dosing regimens of intravenous pembrolizumab versus ipilimumab in patients with advanced melanoma who have not received ipilimumab (but may have had other therapy such as BRAF inhibitors)—found that pembrolizumab provided superior overall survival. ${ }^{2}$ The hazard ratio (HR) for death was 0.69 (95\% confidence interval $[C I]=0.52-0.90)$ for patients dosed with pembrolizumab every 3 weeks as compared with the ipilimumab group. Further, the adverse event rates in the pembrolizumab groups in KN006 were considerably lower. ${ }^{2}$ In addition, a previous trial 
(KEYNOTE-002) found that pembrolizumab improved healthrelated quality of life compared with chemotherapy. ${ }^{3}$

Subsequently, the FDA approved an expanded indication for pembrolizumab (first-line use for patients with advanced melanoma) and the National Comprehensive Cancer Network recommended it as one of the first-line treatments for patients with advanced melanoma in its clinical practice guidelines. ${ }^{4}$

However, there have been no published cost-effectiveness analyses for pembrolizumab among patients with advanced melanoma. When payers make reimbursement/formulary decisions, they consider the overall value of a drug. For this purpose, cost-effectiveness analysis (CEA) has been increasingly used by many reimbursement agencies worldwide. ${ }^{5}$ In the United States, there have also been recent attempts by large health plans to implement value-based formularies that use CEA, which have been successful (e.g., by Premera Blue Cross in 2010). ${ }^{6,7}$ The Institute of Clinical and Economic Review, a nonprofit organization that evaluates evidence on the value of medical tests, treatments, and delivery system innovations, has been receiving increased attention because of its use of a value framework in which CEA is one of the key components. ${ }^{8}$ Further, the use of CEA has been included in the Formulary Leveraged Improved Prescribing project. ${ }^{9}$ CEA models are also included in version 4.0 of the Academy of Managed Care Pharmacy (AMCP) Format for Formulary Submissions. ${ }^{10}$

The objective of this study was to evaluate the long-term cost-effectiveness of pembrolizumab relative to ipilimumab in patients with advanced melanoma previously untreated with ipilimumab from the perspective of a U.S. integrated health system.

\section{Methods}

Using Excel (Microsoft, Redmond, WA), a partitioned survival model was built with 3 mutually exclusive health states: progression-free, postprogression, and death. Patients, modeled after those in the KN006 trial, start in the progressionfree state. The postprogression state occurs after the first progression, as defined in the trial by an independent radiologist and oncologist review using the Response Evaluation Criteria in Solid Tumors (RECIST) version 1.1. ${ }^{11}$ The 2 specific comparators were ipilimumab dosed at $3 \mathrm{mg}$ per kg every 3 weeks (Q3W) for a maximum of 4 doses and pembrolizumab dosed at $2 \mathrm{mg}$ per $\mathrm{kg}$ Q3W to be consistent with the most recent FDA-approved prescribing information and because it has been shown to be clinically equivalent to the dose of $10 \mathrm{mg}$ per $\mathrm{kg}$ Q3W used in the trial in recently published studies. ${ }^{12-14}$ Further, the $2 \mathrm{mg}$ per $\mathrm{kg}$ Q3W dose is typically used in clinical practice in the United States (Unpublished data on file, "Treatment Patterns of Patients with Metastatic Melanoma in the U.S. Community Oncology Clinical Practice" [available upon request from Merck \& Co] and presented abstract at Society for Melanoma Research 2016 Congress $^{15}$ ). Pembrolizumab was given for up to 24 months. Those who were still in progression-free survival at the end of 24 months were eligible to receive up to 12 months of re-induction treatment if they experienced a disease progression within a 2-year follow-up period. The time horizon in the base-case analysis of the model was 20 years, since it captured relevant differences in the tails of the survival curves, although most patients did not survive that long. Adverse events were considered based on rates and disutility scores for adverse events taken from the KN006 trial data and costs from the Centers for Medicare \& Medicaid Services (CMS).

Data used from the KN006 trial were based on 277 patients on pembrolizumab every 3 weeks and 278 patients on ipilimumab. Proportions of patients in each health state were calculated based on actual and projected survival curves for progression-free survival and overall survival. For the progression-free survival curves, Kaplan-Meier estimates from the KN006 trial were used for the first 60 weeks (median follow-up time). After that, the model used parametric survivor functions fitted to the trial data of week 13 and beyond. Week 13 was chosen as the starting point to project long-term survival curves because there was a discontinuity in the Kaplan-Meier curve related to a protocol-driven radiologic scan. Weibull, exponential, log-normal, log logistic, and Gompertz models were assessed by the Akaike Information Criterion (AIC) and the Bayesian Information Criterion (BIC). For ipilimumab, the log-normal parametric function was used to generate progression-free survival curves as it was best according to AIC and BIC. For pembrolizumab, the Weibull and exponential parametric functions had the best AIC and BIC, respectively. Of those, the Weibull distribution was used for the base case based on clinical opinion that the flatter tail would better reflect the long-term benefit of immunotherapy.

For the overall survival curves, the model used the KaplanMeier estimates of pembrolizumab and ipilimumab from the KN006 trial for the first 60 weeks. Ipilimumab overall survival from week 60 to 260 was then extrapolated assuming the same hazard rates as in a long-term ipilimumab study by Schadendorf et al. (2015). ${ }^{16}$ The overall survival curve for pembrolizumab between week 60 and 260 was extrapolated by applying a time-varying HR of pembrolizumab versus ipilimumab, based on a (best-fitting) log-normal function estimated with the overall survival data of pembrolizumab and 
ipilimumab from the trial. Beyond 260 weeks, hazard rates from the American Joint Committee on Cancer melanoma registry were used to estimate overall survival for all patients where background mortality derived from 2011 U.S. life tables was also incorporated because the melanoma registry only reports cancer-related deaths. ${ }^{17}$

The model made a simplifying assumption that best supportive care (which included "no active treatment") was the only subsequent therapy administered after progression for both treatment drugs. This assumption was applied because the trial data did not show a significant difference in postprogression drug use between the 2 arms, and other assumptions would require speculation regarding efficacy from various sequences and durations of drug use.

Clinical data-including progression-free survival and overall survival data, quality of life, and adverse event dataoriginated from the second interim analysis of the ongoing KN006 trial (cut-off date: March 3, 2015). ${ }^{2}$ In addition, longterm survival information for patients treated with ipilimumab and a variety of cost data were identified from the literature and the official CMS websites as described below.

\section{Costs}

This study used drug prices available online in 2015 from Analysource.com. Pembrolizumab was $\$ 4,316$ per 100 -mg vial, and ipilimumab was $\$ 6,659.07$ per $50 \mathrm{mg}^{18}$ For each administration, pembrolizumab was dosed at $2 \mathrm{mg}$ per $\mathrm{kg}$, and ipilimumab was dosed at $3 \mathrm{mg}$ per $\mathrm{kg}$. Drug acquisition costs were calculated in whole vials rounded up at the patient level. Based on the patient weight distribution from U.S. patients in the KN006 trial, the average number of vials was 2.24 of $100 \mathrm{mg}$ for pembrolizumab and $5.92 \mathrm{of} 50 \mathrm{mg}$ for ipilimumab per administration. Ipilimumab patients took the drug every 3 weeks for a total of 4 doses unless the drug was stopped because of unacceptable toxicity or disease progression. The model projected the average number of doses of ipilimumab per patient as 3.3, which was consistent with the trial data.

Pembrolizumab patients in the KN006 trial took the drug every 3 weeks until disease progression, the onset of unacceptable side effects, an investigator's decision to discontinue treatment, withdrawal or patient consent, or 24 months of therapy. Patients who were still in progression-free survival at the end of 24 months were eligible to receive up to 12 months of reinduction treatment if they experienced a disease progression within a 2-year follow-up period. The model projected that $48 \%$ of the patients in progression-free survival would receive re-induction. The projected drug duration over 20 years across all pembrolizumab patients was 11 months (where it was observed to be 5 months at the database-lock time of trial).

Drug administration costs per dose were estimated from rates used in the 2015 Medicare hospital outpatient prospective payment system. ${ }^{19}$ The model also incorporated costs for routine oncology office visits, lab tests, and scans in various health states, as well as a 1-time cost of terminal care to approximate health care cost in the last 3 months of life (for details, see Table 1$) \cdot{ }^{20}$

Noninvestigational (i.e., not lab-related) grade 3-4 adverse events aggregated as categories (e.g., gastrointestinal events) that affected at least $2 \%$ of the patients in at least 1 of the treatment arms were included in the model for both arms. Based on clinical opinion, noninvestigational grade 3-4 adverse events were modeled to result in hospitalization. Hospitalization costs of the specified grade 3-4 adverse events were taken from the Medicare acute inpatient prospective payment system for 2015 based on weighted averages of diagnosis-related group-based hospital payments constructed from the Final Rule Tables used by CMS. ${ }^{21}$ See the Appendix for further detail (available in online article).

\section{Utility Scores}

Utility scores were based on quality of life data collected in KN006 with missing values excluded. The EuroQol (EQ-5D) was administered at visits of cycles 1, 2, 3, 5, 9, and 13 for pembrolizumab and at visits of cycles 1,2 , and 3 , and posttreatment cycles 1 and 5 for ipilimumab. The EQ-5D was also administered at drug discontinuation visits and day 30 safety follow-up visits. Responses to the EQ-5D questionnaire were converted to population-based utility values using a published U.S.-based algorithm. ${ }^{22}$ In considering whether to pool the data across both arms, statistical significance based on a $P$ value of 0.05 was considered.

Mean EQ-5D scores associated with the following time-todeath categories were calculated: 360 days or more, $270-360$ days, 180-270 days, 90-180 days, 30-90 days, and under 30 days to death. ${ }^{23}$ Death was assigned a utility of 0 .

Utility scores associated with patients experiencing grade 3-4 adverse events were also compared with those when patients were not experiencing adverse events. The difference between visits with and without grade 3-4 adverse events were used to estimate the average disutility associated with adverse events. For the base-case analysis, the mean utility decrement for an adverse event from the pooled data analysis as of 0.18 was used, and the duration of the adverse events was conservatively taken to be 4 weeks. Robustness of the results was tested in the 
Utility: mean $(95 \% \mathrm{CI})$

$\geq 360$ days until death

270-360 days until death

180-270 days until death

90-180 days until death

30-90 days until death

$<30$ days until death

PFS

Postprogression

Adverse events

Gastrointesinal disorders

General disorders and administrative site conditions

Metabolism and nutrition disorder

Costs of adverse event management ${ }^{\mathrm{c}}$

Disutility of an adverse event ${ }^{c}$

Drug costs

Unit cost of drug

Mean number of vials per 3 weeks (based on whole vials at the patient level)

Mean administrative cost of drugd

Total drug cost for each dose

Disease management costs ${ }^{\mathrm{e}}$

Management during PFS on treatment

Management during PFS off treatment

Management postprogression

Death-related costs

\begin{tabular}{|l|l|}
\hline & \\
\hline & \\
\hline & \\
\hline & \\
\hline & \\
\hline
\end{tabular}

\begin{tabular}{ll}
0.85 & $(0.84-0.87)$ \\
0.78 & $(0.73-0.83)$ \\
\hline 0.74 & $(0.70-0.78)$ \\
\hline 0.75 & $(0.72-0.79)$ \\
\hline 0.69 & $(0.64-0.74)$ \\
\hline & $0.48(0.35-0.61)$ \\
\hline & $0.78(0.75-0.80)$ \\
\hline
\end{tabular}

a Functions selected based on Akaike Information Criterion and Bayesian Information Criterion for best fit in weeks 13 and beyond from the trial data.

${ }^{b}$ Functions selected based on Akaike Information Criterion and Bayesian Information Criterion for best fit from the trial data.

cAdverse event costs were based on frequencies of noninvestigational grade 3 or higher adverse events that affected at least $2 \%$ of patients in either arm and published CMS cost estimates. ${ }^{19}$ Adverse event disutility was measured by pooling utility scores from patients experiencing an adverse event versus patients in weeks without an adverse event. Adverse events were then modeled as lasting 4 weeks. DRGs for gastrointestinal disorders were 371, 372, and 373, and costs were $\$ 7,159.38$ from CMS estimates; for general disorders and adminstrative site conditions, the DRG codes were 947 and 948, and the costs were \$4,350.38; and for metabolism and nutrition disorder the DRGs were 640 and 641 , and the costs were $\$ 5,352.60 .19$

${ }^{d}$ Drug administration costs are from CMS outpatient cost estimates. ${ }^{21}$ Ipilimumab is given once every 3 weeks for 4 doses, and pembrolizumab is given every 3 weeks until disease progression or 24 months; 48\% of patients in PFS at the end of 2 years are projected to receive a second course for a maximum of 12 months.

¿Disease management costs include oncology office visits, lab tests, scans, and costs from recently published estimates of melanoma-related costs. ${ }^{20}$

$C I=$ confidence interval; $C M S=$ Centers for Medicare $\&$ Medicaid Services; DRG=Diagnosis-Related Group; OS=overall survival; $P F S=$ progression-free survival

sensitivity analysis by varying the duration of adverse events.

For each health state, a specific cost and quality-of-life adjustment weight was assigned for each 1-week cycle to calculate cumulative costs and cumulative quality-adjusted life-years (QALYs). Costs related to adverse events and utility decrements were applied separately to each drug, assuming that the events occurred at the beginning of the study. Costs and health outcomes were discounted at a rate of 3\% per year. The model was used to project costs, life-years, QALYs, and the incremental cost-effectiveness ratio (ICER), expressed as the incremental cost per QALY gained, associated with using pembrolizumab versus ipilimumab in ipilimumab-naive patients.

\section{Sensitivity Analyses}

Numerous scenario analyses were conducted. The analyses examined the impact of several different methodologies used in the extrapolation of the survival (progression-free and overall) curves, versions of the model with different time horizons, and versions with different assumptions of treatment strategy for pembrolizumab. Assigning average utility for each health state before and after progression was also examined.

Deterministic one-way sensitivity analyses were run varying parameters of the extrapolation functions across estimated $95 \%$ CIs, varying utilities by plus or minus $20 \%$, disease management costs across their 95\% CIs, and adverse event 


\section{TABLE 2 Base-Case Results}

\begin{tabular}{|c|c|c|c|}
\hline Model Estimates & Ipilimumab & Pembrolizumab & Difference \\
\hline \multicolumn{4}{|l|}{ Effectiveness (not discounted) } \\
\hline Progression-free life-years & 0.84 & 1.68 & 0.83 \\
\hline Postprogression life-years & 2.98 & 3.29 & 0.30 \\
\hline Life-years & 3.83 & 4.96 & 1.14 \\
\hline QALYs & 3.14 & 4.11 & 0.96 \\
\hline \multicolumn{4}{|l|}{ Effectiveness discounted } \\
\hline Progression-free life-years & 0.78 & 1.54 & 0.76 \\
\hline Postprogression life-years & 2.48 & 2.64 & 0.17 \\
\hline Life-years & 3.26 & 4.18 & 0.93 \\
\hline QALYs & 2.67 & 3.45 & 0.79 \\
\hline \multicolumn{4}{|l|}{ Costs discounted, $\$$} \\
\hline Medication costs & 129,736 & 159,470 & 29,734 \\
\hline Drug administration costs & 1,115 & 4,694 & 3,579 \\
\hline Adverse event costs & 912 & 423 & -489 \\
\hline Additional costs of care & 108,063 & 138,918 & 30,855 \\
\hline Total & 239,826 & 303,505 & 63,680 \\
\hline \multicolumn{4}{|l|}{ Cost-effectiveness, $\$$} \\
\hline Incremental cost per life-year gained & & & 68,712 \\
\hline Incremental cost per QALY gained & & & 81,091 \\
\hline
\end{tabular}

management costs from $50 \%$ to $200 \%$ as best guesses for their potential range given limited available quantitative data. Further, a probabilistic sensitivity analysis was run based on 1,000 sets of simultaneous samples from specified probability distributions of the model inputs. The underlying distributions included a beta distribution using the mean and standard error for the utilities based on the clinical trial and log-normal distributions for the cost inputs using means equal to the base case value and standard errors as reported in the literature or set conservatively at $20 \%$ of the base case value. In addition, the probabilistic sensitivity analyses incorporated uncertainty in the functional forms of the extrapolation functions based on assigning distributions for the key parameters characterizing the extrapolation functions.

\section{Results}

\section{Base-Case Analysis}

Detailed results from the base-case analyses are shown in Table 2 and Figure 1. Patients treated with pembrolizumab spent an average of 1.68 years in the progression-free health state and 3.29 years in the postprogressive health state, resulting in a mean survival estimate of 4.96 years. In the ipilimumab arm, patients spent an average of 0.84 years in the progressionfree health state and 2.98 years in the postprogressive state for a mean survival time of 3.83 years. Hence, pembrolizumab was associated with a gain in mean survival of 1.14 years with much of the gain in the progression-free survival.

In terms of QALYs, pembrolizumab was associated with an average (discounted) gain of 0.79 over ipilimumab. In addition, the base-case model projected a difference of $\$ 63,680$ in the total average per-patient direct cost of treatment with pembrolizumab versus ipilimumab. Therefore, the ICER for pembrolizumab was $\$ 81,091$ per QALY ( $\$ 68,712$ per life-years) over a 20-year time horizon.

\section{Scenario Sensitivity Analyses}

Effect of Changing the Method of Survival Extrapolation. When an exponential function, instead of a Weibull, was used for the progression-free survival of pembrolizumab-treated patients, the ICER was reduced to $\$ 75,445$ per QALY.

Instead of using a time-varying HR function for overall survival beyond week 60, a constant HR of 0.69 (estimated from a stratified Cox proportional-hazards model using the KN006 trial data), ${ }^{2}$ yielded a greater gain in life-years and QALYs and thus improved the ICER to $\$ 69,528$ per QALY. Assuming the hazards of death for patients on the 2 drugs were the same after 60 weeks, the ICER would be $\$ 128,681$ per QALY. We also explored other time points, such as weeks 104, 156, and 208, before removing the treatment effect. In the first period, a fitted log-normal distribution for each arm was used, and then an HR of 1 was applied to pembrolizumab; ipilimumab was assumed to have the same HR as in the Schadendorf data or melanoma registry data. ${ }^{16}$ When the time point of dropping the treatment effect was set at week 104, 156, or 208, the ICER was changed to $\$ 92,159, \$ 82,290$, or $\$ 80,458$ per QALY, respectively.

Also, one could extrapolate using the Schadendorf data and switch to using the registry data at different time periods. ${ }^{16}$ Switching from Schadendorf to registry data at weeks 515 and 208 and keeping everything else as in the base case, the ICERs were $\$ 72,002$ and $\$ 84,610$ per QALY, respectively.

Using log-normal parametric functions for pembrolizumab and ipilimumab for overall survival, the life expectancy over a 20-year time horizon was projected to be 4.51 and 3.29 years (undiscounted), and the ICER was 77,623 per QALY (discounted).

Varying Utility Estimates. Instead of categorizing patient quality of life by time before death, utility can also be grouped into time before and after disease progression. Using this approach, the cost-effectiveness result $(\$ 78,841$ per QALY) was very similar to the base case.

Varying Time Horizons. The time horizon was varied from 10 years to 30 years, which resulted in the ICER varying from 
Progression-Free Survival by IRO Review

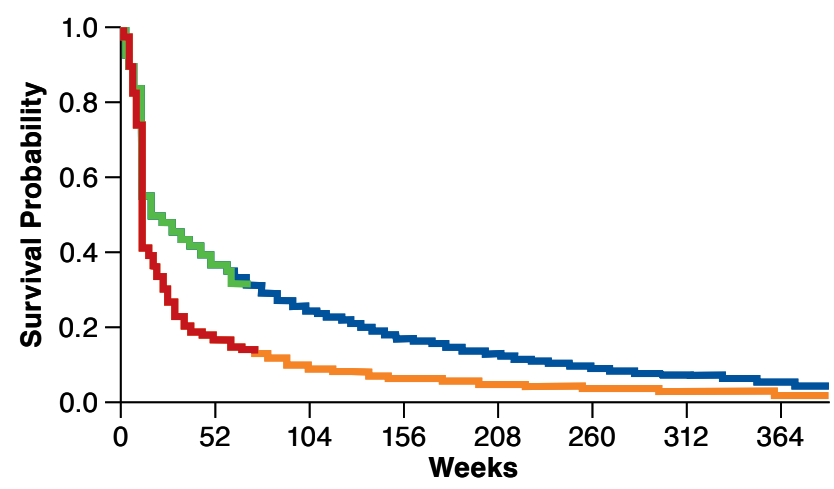

\begin{tabular}{|ll|}
\hline Pembrolizumab & Pembro-KM \\
Ipilimumab & IPI-KM
\end{tabular}

Overall Survival

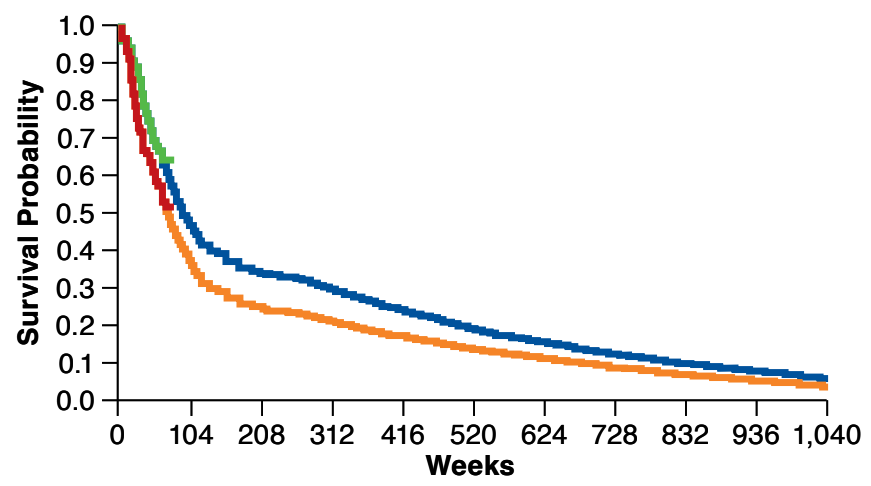

\begin{tabular}{|ll|}
\hline Pembrolizumab & Pembro-KM \\
Ipilimumab & IPI-KM
\end{tabular}

$I P I=$ ipilimumab; IRO= independent review organization; KM = Kaplan-Meier; Pembro= pembrolizumab.

$\$ 94,520$ to $\$ 79,106$. In general, shorter time horizons resulted in higher ICERs, since most of the costs were incurred in the first 2 years of the time horizon, but survival gains continued to be realized after 2 years.

Varying Practice Patterns. Treating pembrolizumab patients until disease progression instead of following the KN006 protocol had a substantial effect on the ICER. Specifically, the ICER increased from $\$ 81,091$ (per the KN006 protocol) to $\$ 212,767$ per QALY if all patients were treated until progression. The ICER was also affected by the assumed proportion of patients receiving a second (12-month maximum) course of treatment. When the proportion of patients who would receive a second course of treatment varied from $0 \%$ to $100 \%$ for those with complete response, partial response, or stable disease at the end of 24 months of treatment, the ICER results ranged from $\$ 59,904$ per QALY to $\$ 103,645$ per QALY.

Varying Adverse Event Duration. The base-case model assumed that the average duration of an adverse event was 4 weeks. However, the duration of adverse events ranged from 0.6 weeks to 14.4 months for various adverse events, as reported in the most recent FDA-approved prescribing information for pembrolizumab. ${ }^{14}$ If the 2 extreme values were used, the ICER changed from the base-case value of $\$ 81,091$ to $\$ 81,163$ and $\$ 79,874$ per QALY, respectively, indicating that a shorter duration of adverse events was a more conservative assumption for pembrolizumab. The ICER result was not sensitive to this assumption.

\section{Deterministic Sensitivity Analyses}

Figure 2 shows the effect of parameter variation on the ICER as derived from the deterministic sensitivity analysis. Model inputs that had the greatest effect on the projected ICER were the proportion of patients who received a second course of pembrolizumab, parameters in the overall survival and progression-free survivor functions, disease management costs, and utility within the time period more than 1 year away from death. Note that the sensitivity analyses related to the parameters of the survival functions are meant to indicate whether the progression-free survival and overall survival data are effective but are unlikely to reflect the actual potential ranges of the progression-free survival and overall survival results. Across all the scenarios used in the sets of one-way deterministic sensitivity analyses, the ICER ranged from $\$ 59,904$ per QALY to $\$ 107,010$ per QALY.

The cost of postprogression used in the model is from a chart review study by Tarhini et al. (2015). ${ }^{20}$ For the sensitivity analysis, monthly cost of cancer patients near death from a 2012 study by Chastek et al. was used as a proxy for postprogression cost, ${ }^{24}$ and the cost-effectiveness result was $\$ 93,185$ per QALY, as the weekly cost increased from $\$ 515$ in the base case to $\$ 1,590$.

\section{Probabilistic Sensitivity Analyses}

Results of Monte Carlo simulations for incremental cost per QALY gained are summarized as a cost-effectiveness acceptability curve in Figure 3. The curve indicates that there were $83 \%, 66 \%$, and $24 \%$ of the simulated ICERs below $\$ 100,000$ 


\section{FIGURE 2 Tornado Diagram for the ICER of Pembrolizumab Versus Ipilimumab}

Proportion of PFS patients who take second course [0, 100] Disease management cost: PFS, off treatment [257, 1,030]

OS HR function (Pem vs. IPI): coefficient [0.1299, 0.6800]

Utility time to death $\geq 360$ days $[0.68,1.02]$

Disease manegement cost: PFS, on treatment $[83,330]$

PFS Pem (13 weeks+): Weibull: intercept [4.3080, 5.2317]

Disease management cost: postprogression [257, 1,030]

PFS Pem (13 weeks+): Weibull: In (scale) [-0.0040, 0.5040]

PFS IPI (13 weeks+): Log-normal: In (scale) [0.2016, 0.6144]

AE cost: IPI [456, 1,824]

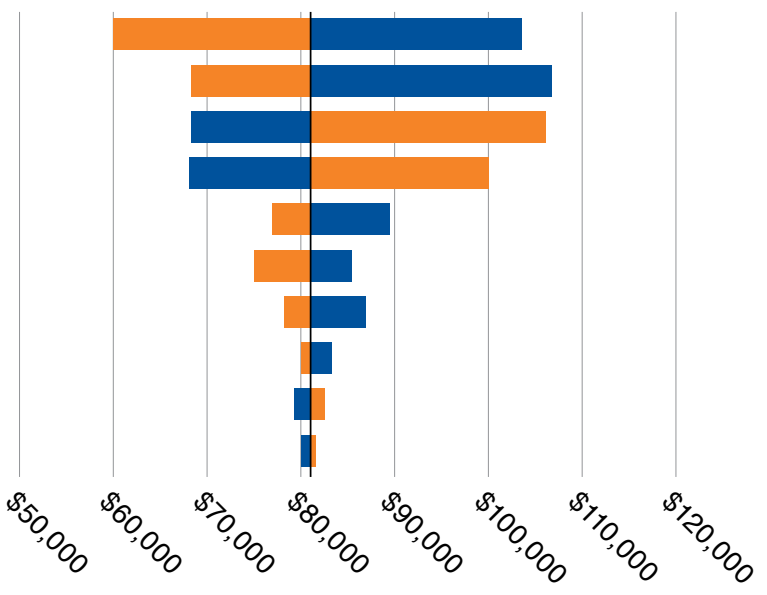

Note: The vertical line in the middle indicates the ICER of the base-case scenario $(\$ 81,091)$. The orange bar indicates the ICER result when the minimum value of the input is used, while the blue bar indicates the ICER result when that maximum value of the input is used.

$A E=$ adverse event; ICER = incremental cost-effectiveness ratio; HR=hazard ratio; IPI=ipilimubam; OS= overall survival; Pem= pembrolizumab; PFS=progression-free survival.

per QALY, \$75,000 per QALY, and \$50,000 per QALY cutoff points, respectively.

\section{Discussion}

Understanding the impact of treatment decisions on costs and outcomes can help decision makers, particularly those in large integrated health systems, improve efficiency and promote value-based treatment choices. Building on the most recently available data, this study provides the first evaluation of the cost-effectiveness of pembrolizumab versus ipilimumab as a treatment for ipilimumab-naïve patients with advanced melanoma. Specifically, the model projected a gain of 0.79 discounted QALYs over ipilimumab and $\$ 63,680$ in discounted per-patient costs of treatment with pembrolizumab versus ipilimumab, with a corresponding ICER of $\$ 81,091$ per QALY over a 20-year time horizon. This value is relatively low compared with many other currently used cancer treatments. ${ }^{25}$ Further, the cost-effectiveness results generally fall under those considered by the Institute for Clinical and Economic Review in its framework, where a threshold of $\$ 100,000-\$ 150,000$ per QALY is used. $^{8}$ The results suggest that, in their treatment-related policy decisions, health systems should favorably view the use of pembrolizumab in patients with advanced melanoma.

The strengths of the model used in this study include use of the KN006 clinical trial data and the best available methods and literature data for extrapolating survival beyond the trial. In addition, the model incorporates published real-world data on relevant cost inputs and EQ-5D-based estimates of utilities for the relevant health states taken directly from the trial.

Further, the results of the model were robust to a variety of sensitivity analyses, including deterministic and probabilistic sensitivity analyses, as well as different scenarios and several variations in the methods used for extrapolating survival past the trial period.

\section{Limitations}

There are, however, limitations to this study that need to be considered. The model does not include a societal perspective. While beyond the scope of our model, and of available data, we anticipate that because pembrolizumab delays progression, considerations of care giving and productivity would make pembrolizumab relatively less costly and thus more cost-effective than in our estimates.

The model was based on a single clinical trial, and treatment effects for pembrolizumab may be different in other patient populations. Also, the trial enrolled patients previously treated with chemotherapy, between $10 \%$ and $15 \%$ of patients, which will become less relevant in real practice. ${ }^{2}$ Further, the cost information comes from published costs by CMS, which may not reflect those experienced by large integrated health systems in the United States.

Much of the data was from the ongoing KNOO6 trial with a median follow-up of 15 months, and data were not available on the re-induction of pembrolizumab at disease progression per the KN006 protocol. ${ }^{2,26}$ It is unclear what percentage of patients 


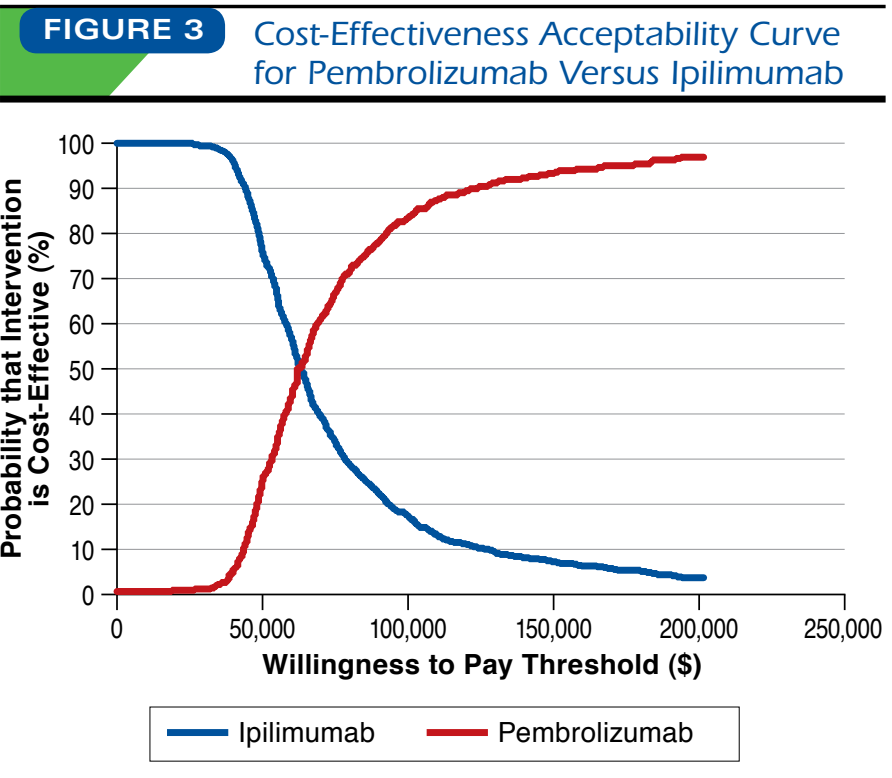

could benefit from this strategy and what the benefit would be. Following the trial protocol, the model projected in the base case that $48 \%$ of the pembrolizumab patients who were in complete response, partial response, or having stable disease would receive an assumed 12-month course of treatment after 2 years, and the model was sensitive to this assumption.

There is also uncertainty regarding whether treatment with pembrolizumab would continue until disease progression. The protocol stipulated that patients in the progression-free state for 2 years must stop the treatment and then re-initiate if there is progression within 2 years. ${ }^{2}$ However, patients in the trial may not have followed the protocol and instead may have opted to stay on treatment indefinitely. If all the patients stayed on pembrolizumab until disease progression, it would have resulted in a significantly higher ICER over a 10- to 20-year time horizon. In clinical practice, treatment patterns may also differ from the trial. However, little data were available to indicate how many patients would haved stayed on treatment past 2 years. Future data on actual treatment patterns can be used to update projections of the cost-effectiveness of pembrolizumab.

In addition, the current model did not consider the possibility that ipilimumab might be re-induced, since it was not allowed in the KN006 trial. Re-induction of ipilimumab would increase the costs of ipilimumab but could improve survival. Hence, the impact on the ICER is uncertain. Unfortunately, the data needed to address this issue will not be available for several years.
Drug duration was modeled as time to progression using RECIST criteria. However, immune-related response criteria (irRC) may be more relevant. ${ }^{27}$ Notably, a recent study by Hodi et al. (2016) using RECIST versus irRC found that RECIST may underestimate the benefit in roughly $15 \%$ of patients in terms of better quality of life and lower disease management costs in the progression-free state. ${ }^{27}$ Using irRC may also mean longer drug duration. Nonetheless, with a 24-month cap, this likely did not meaningfully affect the cost-effectiveness results.

The results are sensitive to long-term survival results, which at this point have to be extrapolated, since limited data are available on the long-term survival of pembrolizumab patients. Although rigorous methodological approaches have been applied and several sensitivity analyses were performed to assess potential variance in the results, actual survival patterns may be different.

The model assumed that best supportive care was the only later-line therapy for patients following progression. More data are needed to see the pattern of postprogression drug use, which could affect the costs and outcomes postprogression and thus the ICER. However, this was beyond the scope of the original research question, which was the comparison of pembrolizumab and ipilimumab, all else being equal.

The utility values may not have fully captured patient quality of life during the entire postprogression phase, since they were generally collected shortly after progression. However, the base-case utilities incorporating time to death and more conventional approaches yielded similar results.

Finally, the model projected costs and outcomes pertinent to the choice between pembrolizumab and ipilimumab. There remain important considerations towards other treatments, such as the recently developed BRAF inhibitors, as relevant comparators. ${ }^{28}$

\section{Conclusions}

The KN006 trial established the clinical benefit of pembrolizumab as a new standard of care for ipilimumab-naïve patients. The model developed for this study indicated that pembrolizumab was likely to be a cost-effective option from the perspective of a U.S. integrated health system over a 20-year time horizon. However, there was substantial uncertainty in the extended use of pembrolizumab in patients remaining in the progression-free state beyond 2 years that had the potential to substantially increase the costs of care. 


\section{Authors}

JINGSHU WANG, PhD; JAMES PELLISSIER, PhD; and RUIFENG $\mathrm{XU}, \mathrm{PhD}$, Predictive \& Economic Modeling, Merck Research Laboratories. FRANK XIAOQING LIU, PhD, Outcomes ResearchOncology, Center for Observational and Real World Evidence (CORE), Merck Research Laboratories, Merck \& Co, North Wales, Pennsylvania, and KENDALL STEVINSON, MD, MPH, Outcomes Research, Merck Research Laboratories, Merck \& Co., Lebanon, New Jersey. BARTOSZ CHMIELOWSKI, MD, PhD, Jonsson Comprehensive Cancer Center, Division of Hematology and Medical Oncology, University of California, Los Angeles.

AUTHOR CORRESPONDENCE: Frank Xiaoqing Liu, PhD, Director, Outcomes Research - Oncology, Center for Observational and Real World Evidence (CORE), Merck Research Laboratories, 351 N. Sumneytown Pike, North Wales, PA 19454-1099. Tel.: 267.305.1265; E-mail: Xiaoqing.liu@merck.com.

\section{DISCLOSURES}

This study was supported by funding from Merck \& Co., which reviewed and approved the manuscript before journal submission. Wang, Pellissier, $\mathrm{Xu}$, Stevinson, and Liu are employees of, and own stock in, Merck \& Co. Chmielowski has served as a paid consultant for Merck \& Co. and received a consultant fee for clinical input in connection with this study. Chmielowski also reports receiving advisory board and speaker bureau fees from multiple major pharmaceutical companies.

Wang led the modeling and writing of the manuscript. Chmielowski, Xu, Stevinson, and Pellissier contributed substantially to the modeling design and methodology. Liu led the data collection work and contributed substantially to writing the manuscript. In conducting the analysis and writing the manuscript, the authors followed Merck publication polices and the "costeffectiveness analysis alongside clinical trials-good research practices" and the CHEERS reporting format as recommended by the International Society for Pharmacoeconomics and Outcomes Research.

\section{ACKNOWLEDGMENTS}

Surrey Walton of Second City Outcomes Research helped draft and revise the manuscript.

\section{REFERENCES}

1. Robert C, Ribas A, Wolchok JD, et al. Anti-programmed-death-receptor-1 treatment with pembrolizumab in ipilimumab-refractory advanced melanoma: a randomised dose-comparison cohort of a phase 1 trial. Lancet. 2014;384(9948):1109-17.

2. Robert C, Schachter J, Long GV, et al. Pembrolizumab versus ipilimumab in advanced melanoma. N Engl J Med. 2015;372(26):2521-32.

3. Schadendorf D, Dummer R, Hauschild A, et al. Health-related quality of life in the randomised KEYNOTE-002 study of pembrolizumab versus chemotherapy in patients with ipilimumab-refractory melanoma. Eur J Cancer. 2016;67:46-54.
4. Coit DG, Thompson JA, Algazi A, et al. Melanoma, version 2. 2016, NCCN clinical practice guidelines in oncology. J Natl Compr Canc Netw. 2016;14(4):450-73.

5. Brousselle A, Lessard C. Economic evaluation to inform health care decision-making: promise, pitfalls and a proposal for an alternative path. Soc Sci Med. 2011;72(6):832-39.

6. Sullivan SD, Yeung K, Vogeler C, et al. Design, implementation, and first-year outcomes of a value-based drug formulary. J Manag Care Spec Pharm. 2015;21(4):269-75. Available at: http://www.jmcp.org/doi/10.18553/ jmcp.2015.21.4.269.

7. Yeung K, Basu A, Hansen RN, et al. Impact of a value-based formulary on medication utilization, health services utilization, and expenditures. Med Care. August 30, 2016. [Epub ahead of print]

8. Institute for Clinical and Economic Review. ICER value assessment framework 1.0 to 2.0. 2016. Available at: http://icer-review.org/wp-content/ uploads/2016/02/Value-Assessment-Framework-slides-for-July-29-webinarFINAL.pdf. Accessed January 18, 2017.

9. Schiff GD, Galanter WL, Duhig J, et al. A prescription for improving drug formulary decision making. PLoS Med. 2012;9(5):1-7.

10. Academy of Managed Care Pharmacy. The AMCP format for formulary submissions. Version 4.0. April 2016. Available at: http://www.amcp.org/ FormatV4/. Accessed January 11, 2017.

11. Eisenhauer EA, Therasse P, Bogaerts J, et al. New response evaluation criteria in solid tumours: Revised RECIST guideline (version 1.1). Eur J Cancer. 2009;45(2):228-47.

12. Ribas A, Puzanov I, Dummer R, et al. Pembrolizumab versus investigator-choice chemotherapy for ipilimumab-refractory melanoma (KEYNOTE-002): a randomised, controlled, phase 2 trial. Lancet Oncol. 2015;16(8):908-18.

13. Ribas A, Hamid O, Daud A, et al. Association of pembrolizumab with tumor response and survival among patients with advanced melanoma. JAMA. 2016;315(15):1600-09.

14. KEYTRUDA (pembrolizumab) for injection, for intravenous use. Merck $\&$ Co. Revised October 2015. Available at: www.accessdata.fda.gov/drugsatfda_docs/label/2015/125514s005lbl.pdf. Accessed January 11, 2017.

15. Cowey CL, Liu FX, Black-Shinn J, et al. Real-world utilization and outcomes of pembrolizumab for the treatment of patients with advanced melanoma in U.S. community oncology practice: a retrospective observational study. Poster presented at: Society for Melanoma Research 2016 Congress; November 6-9, 2016; Boston, MA. Available at: https://www.melanomacongress.com/docs/2016_abstracts.pdf [Page 25]. Accessed January 18, 2017.

16. Schadendorf D, Hodi FS, Robert C, et al. Pooled analysis of long-term survival data from phase ii and phase III trials of ipilimumab in unresectable or metastatic melanoma. J Clin Oncol. 2015;33(17):1889-94.

17. Balch CM, Buzaid AC, Soong SJ, et al. Final version of the American Joint Committee on Cancer staging system for cutaneous melanoma. J Clin Oncol. 2001;19(16):3635-48.

18. Analysource. Suite of drug pricing services. 2016. Available at: www. analysource.com. Accessed January 11, 2017.

19. Center for Medicare \& Medicaid Services. Costs for hospital outpatient services by HCPCS code for CY 2015. Available at: https://www.cms.gov/ apps/ama/license.asp?file=/Medicare/Medicare-Fee-for-Service-Payment/ HospitalOutpatientPPS/Downloads/CY-2015-OPPS-CN-Cost-Statistics-Files. zip. Accessed January 11, 2017. 
20. Tarhini A, Corman SL, Rao S, et al. Healthcare resource utilization and associated costs in patients with advanced melanoma receiving first-line ipilimumab. J Cancer Ther. 2015;6:833-40.

21. Center for Medicare \& Medicaid Services. FY 2015 final rule tables (Tables 1, 5, and 7). Available at: https://www.cms.gov/Medicare/MedicareFee-for-Service-Payment/AcuteInpatientPPS/FY2015-IPPS-Final-Rule-HomePage-Items/FY2015-Final-Rule-Tables.html?DLPage $=1 \&$ DLEntries $=10 \& D L S o$ $\mathrm{rt}=0 \& D L S o r t D i r=a s c e n d i n g$. Accessed January 11, 2017.

22. Shaw JW, Johnson JA, Coons SJ. U.S. valuation of the EQ-5D health states: development and testing of the Dl valuation model. Med Care. 2005;43(3):203-20.

23. Hatswell AJ, Pennington B, Pericleous L, Rowen D, Lebmeier M, Lee D. Patient-reported utilities in advanced or metastatic melanoma, including analysis of utilities by time to death. Health Qual Life Outcomes. 2014;12:140.
24. Chastek B, Harley C, Kallich J, et al. Health care costs for patients with cancer at the end of life. J Oncol Pract. 2012;8(6):75s-80s.

25. Howard DH, Bach PB, Berndt ER, Conti RM. Pricing in the market for anticancer drugs. J Econ Perspect. 2015;29(1):139-62. Available at: https:// www.aeaweb.org/articles?id=10.1257/jep.29.1.139. Accessed January 18, 2017.

26. Protocol for: Robert C, Schachter J, Long GV, et al. Pembrolizumab versus ipilimumab in advanced melanoma. N Engl J Med. 2015;372(26):252132. Available at: http://www.nejm.org/doi/suppl/10.1056/NEJMoal503093/ suppl_file/nejmoal503093_protocol.pdf. Accessed January 11, 2017.

27. Hodi FS, Hwu WJ, Kefford R, et al. Evaluation of immune-related response criteria and RECIST vl.1 in patients with advanced melanoma treated with pembrolizumab. J Clin Oncol. 2016;34(13):1510-17.

28. Jarkowski A, Norris L, Trinh VA. Controversies in the management of advanced melanoma: "gray" areas amid the "black and blue." Ann Pharmacother. 2014:48(11):1456-68. 
Cost-Effectiveness of Pembrolizumab Versus Ipilimumab in

Ipilimumab-Naïve Patients with Advanced Melanoma in the United States

\section{APPENDIX Unit Costs}

\begin{tabular}{|c|c|c|c|}
\hline \multirow{2}{*}{\multicolumn{4}{|c|}{ Drug administration costs for Medicare for outpatient PPS 2015, $\$$}} \\
\hline & & & \\
\hline Administration cost for first hour of chemotherapy infusion & 285.11 & CPT code 96413 & $\mathrm{CMS}^{19}$ \\
\hline Administration cost for additional hour of chemotherapy infusion & 53.54 & CPT code 96415 & $\mathrm{CMS}^{19}$ \\
\hline $\begin{array}{l}\text { Administration cost per hour for subsequent chemotherapy infusion } \\
\text { (applies to second chemotherapy for combination chemotherapy) }\end{array}$ & 53.54 & CPT code 96417 & $\mathrm{CMS}^{19}$ \\
\hline Outpatient physician visit & 96.25 & CPT code 99215 & $\mathrm{CMS}^{19}$ \\
\hline \multicolumn{4}{|l|}{ Disease management costs, $\$$} \\
\hline Management during PFS on treatment per week & $165 \pm 748$ & $\begin{array}{l}\text { Cost of treatment from Tarhini et al. adjusted } \\
\text { to } 2015 \text { using } 2.6 \% \text { inflation }\end{array}$ & Tarhini et al. ${ }^{20}$ \\
\hline Management during PFS off treatment per week & $515 \pm 1,132$ & $\begin{array}{l}\text { Cost of off treatment from Tarhini et al. } \\
\text { adjusted to } 2015 \text { using } 2.6 \% \text { inflation }\end{array}$ & Tarhini et al. 20 \\
\hline Management postprogression per week & $515 \pm 1,132$ & $\begin{array}{l}\text { Cost of off treatment from Tarhini et al. } \\
\text { adjusted to } 2015 \text { using } 2.6 \% \text { inflation }\end{array}$ & Tarhini et al. ${ }^{20}$ \\
\hline Death-related costs (one-off) & $15,769 \pm 28,899$ & $\begin{array}{l}\text { From Tarhini et al. adjusted to } 2015 \text { using } \\
2.6 \% \text { inflation }\end{array}$ & Tarhini et al. ${ }^{20}$ \\
\hline \multicolumn{4}{|l|}{ Hospitalization costs for adverse events, ${ }^{\text {a }} \$$} \\
\hline Gastrointestinal disorders (grade $3+$ ) & $7,159.38$ & DRG codes 371-372-373 & $\mathrm{CMS}^{21}$ \\
\hline General disorders and administration site condition (grade 3+) & $4,350.38$ & DRG codes 947-948 & $\mathrm{CMS}^{21}$ \\
\hline Metabolism and nutrition disorder (grade 3+) & $5,352.60$ & DRG codes $640-641$ & $\mathrm{CMS}^{21}$ \\
\hline
\end{tabular}

aAdverse event costs were based on frequencies of grade 3 or higher adverse events that affected at least $2 \%$ of patients in either arm and published CMS cost estimates. ${ }^{21}$

The cost estimates involved standard costs and multipliers weighted by frequencies of severity seen in the CMS data (using Tables 1, 5, and 7).21

CMS = Centers for Medicare \& Medicaid Services; CPT = Current Procedural Terminology; DRG=Drug-Related Group; PFS=progression-free survival; PPS = prospective payment system. 